\title{
POLAR LINKINGS, INTERSECTIONS, AND WEIL PAIRING
}

\author{
Boris KHESIN and Alexei ROSLY
}

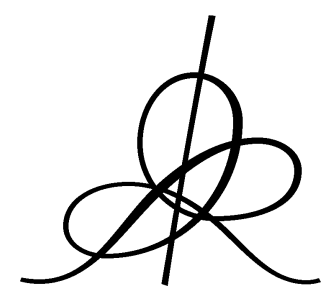

Institut des Hautes Études Scientifiques

35 , route de Chartres

91440 - Bures-sur-Yvette (France)

Août 2003

IHES $/ \mathrm{M} / 03 / 53$ 


\title{
Polar Linkings, Intersections, and Weil Pairing
}

\author{
Boris Khesin* and Alexei Rosly ${ }^{\dagger}$
}

July 29,2003

\begin{abstract}
Polar homology and linkings arise as natural holomorphic analogues in algebraic geometry of the homology groups and links in topology. For complex projective manifolds the polar $k$-chains are subvarieties of complex dimension $k$ with meromorphic forms on them, while the boundary operator is defined by taking the polar divisor and the Poincaré residue on it. We also define the corresponding analogues for the intersection and linking numbers of complex submanifolds, and show that they have the properties similar to those of the corresponding topological objects. Finally, we establish the relation between the holomorphic linking and the Weil pairing of functions on a complex curve and its higher-dimensional counterparts.
\end{abstract}

\section{Introduction.}

Polar homology groups for complex projective manifolds can be regarded as a complex version of singular homology groups in topology. The idea of such a geometric analogue of topological homology comes from thinking of the Dolbeault (or $\bar{\partial})$ complex of $(0, k)$ forms on a complex manifold as an obvious analogue of the de Rham complex of $k$-forms on a smooth manifold. This poses an immediate question: "What is the analogue of the chain complex relevant to the context of complex manifolds?"

In this paper we propose the complex of "polar chains," which consists of algebraic subvarieties and meromorphic forms on them, whose poles are of the first order only. The boundary operator is taking the polar divisor and the Poincaré residue of the corresponding form. Such a complex can be viewed as a subcomplex of the Cousin complex in algebraic geometry. An advantage of considering "polar chains" is that this allows one to use the analogy between the boundary operator in topology and the residue in algebraic geometry in a very direct way. In particular, one can define analogues for the intersection and linking numbers of complex submanifolds mimicking properties of the corresponding topological notions.

Some features of the above analogy between boundaries and residues can also be found in the recent literature. In particular, this correspondence leads to an explicit

\footnotetext{
*Department of Mathematics, University of Toronto, Toronto, ON M5S 3G3, Canada; e-mail: khesin@math.toronto.edu

${ }^{\dagger}$ Institute of Theoretical and Experimental Physics, B.Cheremushkinskaya 25, 117259 Moscow, Russia; e-mail: rosly@heron.itep.ru
} 
construction of a group cocycle for the double loop groups [FK]. It is useful in the study of the Poisson structure on the moduli spaces of holomorphic bundles on complex surfaces (see the description of Lagrangian submanifolds in [DT, Th] and that of symplectic leaves in $[\mathrm{KR}])$. Moreover, the approach of Donaldson and Thomas [DT] of transferring differential geometric constructions into the context of complex analytic (or algebraic) geometry could lead one to a "complexification of geometry" in a sense similar to the "complexification of topology" pursued here.

Below we mention briefly one of the main motivations, which come from mathematical physics: to quantify a complex analogue of the Chern-Simons gauge theory on a CalabiYau threefold suggested in [Wit2]. The latter context leads us immediately to a search for a proper holomorphic analogue of the linking number (cf., also [FT, Th]). Below we introduce the notions of holomorphic intersection and linking in terms of polar cycles (i.e., complex submanifolds equipped with holomorphic volume forms). The language of polar chains suggested in this paper provides a convenient framework for the description of the (co)homological structure underlying the above problems.

1.1 Motivation and holomorphic linking. The classical linking number arises in the study of quantum theory associated with the abelian Chern-Simons functional

$$
C S(A)=\int_{M} A \wedge d A
$$

defined on smooth 1-forms $\{A\}$ on a real 3-fold $M$, [Sch, Pol]. (The non-abelian ChernSimons theory leads to higher link invariants, such as Jones polynomials, [Wit1].)

A holomorphic analogue of the Chern-Simons theory on a Calabi-Yau three-dimensional manifold $X$ (with a holomorphic 3 -form $\mu$ ) was suggested in [Wit2]. In the simplest abelian case, the action functional is

$$
C S_{\mu}(A)=\int_{X} A^{01} \wedge \bar{\partial} A^{01} \wedge \mu
$$

where $A^{01}$ is a smooth $(0,1)$-forms on $X$. In the latter case one can consider the following natural observables, analogs of Wilson loops: $\int_{C} \alpha \wedge A^{01}$, where $C$ is a complex curve in the 3 -fold $X$ and $\alpha$ is a holomorphic 1-form on $C$. The correlator of two such observables in this quadratic field theory is given by the following expression:

$$
<\int_{C_{1}} \alpha_{1} \wedge A^{01} \cdot \int_{C_{2}} \alpha_{2} \wedge A^{01}>=\sum_{P \in C_{1} \cap S_{2}} \frac{\alpha_{1}(P) \wedge \beta_{2}(P)}{\mu(P)} .
$$

Here $S_{2}$ is a complex surface containing the curve $C_{2}$ and equipped with a meromorphic 2 -form $\beta_{2}$, such that $\operatorname{div}_{\infty} \beta_{2}=C_{2}$ and $\operatorname{res}_{C_{2}} \beta_{2}=\alpha_{2}$. Such a sum over the intersection points of $C_{1}$ and $S_{2}$ can be thought of as a holomorphic linking number of two nonintersecting complex curves $C_{1}$ and $C_{2}$, equipped with holomorphic 1-forms $\alpha_{i}$ in the Calabi-Yau manifold $X$ :

$$
\ell k_{\mu}\left(\left(C_{1}, \alpha_{1}\right),\left(C_{2}, \alpha_{2}\right)\right):=\sum_{P \in C_{1} \cap S_{2}} \frac{\alpha_{1}(P) \wedge \beta_{2}(P)}{\mu(P)} .
$$


Recall that the topological linking number of two closed curves in a real 3-fold is defined as the intersection of the first curve with a surface spanning the other curve. One can show that the expression above does not depend on the choice of $\left(S_{2}, \beta_{2}\right)$, a holomorphic analog of a spanning surface, and it has certain invariance properties mimicking those of the classical linking number in this holomorphic situation.

1.2 Holomorphic intersections. On the way we have implicitly defined a complex analogue of the intersection number in topology. To make it explicit, let $(X, \mu)$ be a complex manifold equipped with a meromorphic volume form $\mu$ without zeros. Consider two complex submanifolds $A$ and $B$ of complimentary dimensions that intersect transversely in $X$ and are endowed with volume forms $\alpha$ and $\beta$ on the corresponding submanifolds. Then the holomorphic intersection number is defined by the formula

$$
\langle(A, \alpha) \cdot(B, \beta)\rangle=\sum_{P \in A \cap B} \frac{\alpha(P) \wedge \beta(P)}{\mu(P)} .
$$

At every intersection point $P$, the ratio in the right-hand-side is the "comparison" of the orientations of the "cycles" $(A, \alpha)$ and $(B, \beta)$ at that point with the orientation of the ambient manifold. This is a straightforward analogue of the use of mutual orientation of cycles in the definition of the topological intersection number. Note, that in the holomorphic case the intersection number does not have to be an integer. (Rather, it is a holomorphic function of the "parameters" $(A, \alpha),(B, \beta)$ and $(X, \mu)$.

1.3 Holomorphic orientation and boundary operator. The above consideration prompts us to consider a top degree holomorphic form on a complex manifold as manifold's "holomorphic orientation." Furthermore, a pair $(W, \omega)$, which consists of a $k$ dimensional submanifold $W$ equipped with a meromorphic top degree form $\omega$ (with first order poles on a smooth hypersurface $V$ ) will be thought of as an analogue of a compact oriented submanifold with boundary.

Below we start by defining a homology theory in which the pairs $(W, \omega)$ will play the role of $k$-chains. The corresponding boundary operator assumes the form $\partial(W, \omega)=$ $2 \pi i(V$, res $\omega)$, where $V$ is the polar set of the $k$-form $\omega$, while res $\omega$ is the $(k-1)$-form on $V$, the Poincare residue of $\omega$. Note, that in the situation under consideration, when the polar set $V$ of the form $\omega$ is a smooth $(k-1)$-dimensional submanifold in a smooth $k$-dimensional $W$, the induced "orientaion" on $V$ is given by a regular $(k-1)$-form res $\omega$. This means that $\partial(V$, res $\omega)=0$, or the boundary of a boundary is zero. The latter will be the source of the identity $\partial^{2}=0$ in the homology theory discussed below. We shall call it the polar homology.

1.4 Example. To illustrate this notion, find the polar homology groups $H P_{k}$ of a complex projective curve $X$. In this (and in any) case, all the 0 -chains are cycles. Let $(P, a)$ and $(Q, b)$ be two 0 -cycles, where $P, Q$ are points on $X$ and $a, b \in \mathbb{C}$. They are polar homologically equivalent iff $a=b$. Indeed, $a=b$ is necessary and sufficient for the existence of a meromorphic 1-form $\alpha$ on $X$, such that $\operatorname{div}_{\infty} \alpha=P+Q$ and $\operatorname{res}_{P} \alpha=2 \pi i a, \operatorname{res}_{Q} \alpha=-2 \pi i b$. (The sum of all residues of a meromorphic differential on a projective curve is zero by the Cauchy theorem.) Then, we can write in terms of polar chain complex (to be defined in detail in Sect. 2) that $(P, a)-(Q, a)=\partial(X, \alpha)$. Thus, $H P_{0}(X)=\mathbb{C}$. 
As to polar 1-cycles, these correspond to all possible holomorphic 1-forms on $X$. On the other hand, there are no 1-boundaries, since there are no polar 2-chains in $X$. Hence $H P_{1}(X) \cong \mathbb{C}^{g}$, where $g$ is the genus of the curve $X$. (In particular, the polar Euler characteristics of $X$ equals $1-g$ and coincides with its holomorphic Euler characteristics.)

Similar considerations show that for any $n$-dimensional $X$ we have $H_{n}(X)=$ $H^{0}\left(X, \Omega_{X}^{n}\right)$ and, if $X$ is connected, also $H P_{0}(X)=\mathbb{C}$.

1.5 Pairing to smooth forms and the Cauchy-Stokes formula. There is a pairing between polar chains and smooth differential forms on a manifold: For a polar $k$-chain $(W, \omega)$ and any $(0, k)$-form $u$ such a pairing is given by the integral

$$
\langle(W, \omega), u\rangle=\int_{W} \omega \wedge u
$$

In other words, the polar chain $(W, \omega)$ defines a current on $X$ of degree $(n, n-k)$, where $n=\operatorname{dim} X$. This pairing descends to (co)homology classes by virtue of the following Cauchy-Stokes formula.

Consider a meromorphic $k$-form $\omega$ on $W$ having first order poles on a smooth hypersurface $V \subset W$. Let the $\operatorname{smooth}(0, k)$-form $u$ on $X$ be $\bar{\partial}$-exact, that is $u=\bar{\partial} v$ for some $(0, k-1)$-form $v$ on $X$. Then

$$
\int_{W} \omega \wedge \bar{\partial} v=2 \pi i \int_{V} \operatorname{res} \omega \wedge v
$$

We shall exploit this straightforward generalization of the Cauchy formula as a complexified analogue of the Stokes theorem. Note that the Cauchy-Stokes formula defines the pairing between the polar homology groups of a complex manifold $X$ and the Dolbeault cohomology groups $H_{\bar{\partial}}^{0, k}(X)$. In a separate paper [KRT] with $\mathrm{R}$. Thomas we showed that this pairing defines an isomorphism of the corresponding groups, see Section 3.

1.6 Remark. Most of the above discussion extends to polar chains $(A, \alpha)$ where the meromorphic $p$-form $\alpha$ is not necessarily of top degree, that is $0 \leqslant p \leqslant k$, where $k=\operatorname{dim}_{\mathbb{C}} A$. To define the boundary operator we have to restrict ourselves to the meromorphic forms with logarithmic singularities. The corresponding polar homology groups enumerated by two indices $k$ and $p(0 \leqslant p \leqslant k)$ turn out to interpolate between the Dolbeault groups and the groups of algebraic cycles on a complex projective manifold, see Remark 3.5 below).

\section{Polar homology.}

Here we define a homological complex based on the notion of the polar boundary. The construction is analogous to the definition of homology of a topological space with replacement of continuous maps by complex analytic ones. The notion of the boundary (of a simplex or a cell) is replaced by the Poincaré residue of a meromorphic differential form. 


\section{2a. Preliminaries: Residue and push-forward.}

The Poincaré residue is a higher-dimensional generalization of the classical Cauchy residue, where the residue at a point in a domain of one complex variable is generalized to the residue at a hypersurface.

Let $M$ be an $n$-dimensional complex manifold and $\omega$ be a meromorphic $n$-form on $M$ which is allowed to have first order poles on a smooth hypersurface $V$. Then, the form $\omega$ can be locally expressed as

$$
\omega=\frac{\varrho \wedge d z}{z}+\varepsilon
$$

where $z=0$ is a local equation of $V$ and $\varrho$ (respectively, $\varepsilon$ ) is a holomorphic $(n-1)$-form (resp., $n$-form). Then the restriction $\left.\varrho\right|_{V}$ is a well-defined holomorphic $(n-1)$-form on $V$.

Definition 2.1 The Poincaré residue of the $n$-form $\omega$ is the following $(n-1)$-form on $V$

$$
\operatorname{res} \omega:=\left.\varrho\right|_{V}
$$

2.2 This definition can be readily extended to the case of normal crossing divisors. Let $V$ be a normal crossing divisor in $M$, i.e., $V=\cup_{i} V_{i}$ has only smooth components $V_{i}$ (each entering with multiplicity one) that intersect generically. Suppose that the meromorphic $n$-form $\omega$ in $M$ has the first order poles on $V$. Analogously to the Definition 2.1 one can define a residue at each component $V_{i}$. The resulting $(n-1)$-forms $\operatorname{res}_{V_{j}} \omega$ are then meromorphic and have first order poles at the pairwise intersections $V_{i j}=V_{i} \cap V_{j}$ One can now consider the repeated Poincaré residue at $V_{i j}$. Representing $\omega$ as $\omega=\rho \wedge \frac{d z_{i}}{z_{i}} \wedge \frac{d z_{j}}{z_{j}}$, where $z_{i}=0$ and $z_{j}=0$ are local equations of the components $V_{i}$ and $V_{j}$ respectively one finds that

$$
\operatorname{res}_{i, j} \omega:=\operatorname{res}_{V_{i j}}\left(\operatorname{res}_{V_{j}} \omega\right)=\operatorname{res}_{z_{i}=0}\left(\operatorname{res}_{z_{j}=0} \rho \wedge \frac{d z_{i}}{z_{i}} \wedge \frac{d z_{j}}{z_{j}}\right)=\left.\rho\right|_{V_{i j}} .
$$

Note that the repeated residue differs by sign for different order of the components: $\operatorname{res}_{i, j} \omega=-\operatorname{res}_{j, i} \omega$.

2.3 For a finite covering $f: X \rightarrow Y$ and a function $\varphi$ on $X$ one can define its pushforward, or the trace, $f_{*} \varphi$ as a function on $Y$ whose value at a point is calculated by summing over the preimages taken with multiplicities. The operation $f_{*}$ can be generalized to $p$-forms and to the maps $f$ which are only generically finite.

Suppose that $f: X \rightarrow Y$ is a proper, surjective holomorphic mapping where both $X$ and $Y$ are smooth complex manifolds of the same dimension $n$. The push-forward (or, trace) map is a mapping

$$
f_{*}: \Gamma\left(X, \Omega_{X}^{p}\right) \rightarrow \Gamma\left(Y, \Omega_{Y}^{p}\right) .
$$

To define it one notes that $f$ is finite unramified covering away from a hypersurface in $Y$. Thus $f$ is locally an isomorphism of neighborhoods, and the push-forward form is defined by summing the image forms from all the leaves. The form obtained extends to the whole of $Y$. 
The push-forward map is also defined for meromorphic forms, $f_{*}: \Gamma\left(X, \mathcal{M}^{p}\right) \rightarrow$ $\Gamma\left(Y, \mathcal{M}^{p}\right)$. Furthermore, the operations of push-forward and residue commute.

\section{2b. Polar chains and boundary.}

In this section we deal with complex projective varieties, i.e., closed subvarieties of a complex projective space. (In this setting the complex analytic considerations are equivalent to algebraic ones.) By a smooth projective variety we always understand a smooth and connected one. For a smooth variety $M$ of dimension $n$, we denote by $K_{M}$ the sheaf of regular $n$-forms on $M$.

The space of polar $k$-chains for a complex projective variety $X, \operatorname{dim} X=n$, will be defined as a $\mathbb{C}$-vector space with certain generators and relations.

Definition 2.4 The space of polar $k$-chains $\mathcal{C}_{k}(X)$ is a vector space over $\mathbb{C}$ defined as the quotient $\mathcal{C}_{k}(X)=\hat{\mathcal{C}}_{k}(X) / \mathcal{R}_{k}$, where the vector space $\hat{\mathcal{C}}_{k}(X)$ is freely generated by the triples $(A, f, \alpha)$ described in (i),(ii),(iii) and $\mathcal{R}_{k}$ is defined as relations (R1),(R2),(R3) imposed on the triples.

(i) $A$ is a smooth complex projective variety, $\operatorname{dim} A=k$;

(ii) $f: A \rightarrow X$ is a holomorphic map of projective varieties;

(iii) $\alpha$ is a rational $k$-form on $A$ with first order poles on $V \subset A$, where $V$ is a normal crossing divisor in $A$, i.e., $\alpha \in \Gamma\left(A, K_{A}(V)\right)$.

The relations are:

(R1) $\lambda(A, f, \alpha)=(A, f, \lambda \alpha)$

(R2) $\sum_{i}\left(A_{i}, f_{i}, \alpha_{i}\right)=0$ provided that $\sum_{i} f_{i *} \alpha_{i} \equiv 0$, where $\operatorname{dim} f_{i}\left(A_{i}\right)=k$ for all $i$ and the push-forwards $f_{i *} \alpha_{i}$ are considered on the smooth part of $\cup_{i} f_{i}\left(A_{i}\right)$;

(R3) $(A, f, \alpha)=0$ if $\operatorname{dim} f(A)<k$.

2.5 Remark. By definition, $\mathcal{C}_{k}(X)=0$ for $k<0$ and $k>\operatorname{dim} X$.

The relation (R2), in particular, represents additivity with respect to $\alpha$, that is

$$
\left(A, f, \alpha_{1}\right)+\left(A, f, \alpha_{2}\right)=\left(A, f, \alpha_{1}+\alpha_{2}\right) .
$$

Here we make no difference between a triple and its equivalence class. In particular, if the polar divisor $\operatorname{div}_{\infty}\left(\alpha_{1}+\alpha_{2}\right)$ is not normal crossing, one can replace $A$ by an appropriate blow-up, by the Hironaka theorem, where the pull back of $\alpha_{1}+\alpha_{2}$ is already admissible.

This way, the relation (R2) allows us, in particular, to refer to prime polar chains as pairs replacing a triple $(A, f, \alpha)$ by a pair $(\hat{A}, \hat{\alpha})$, where $\hat{A}=f(A) \subset X, \hat{\alpha}$ is defined only on the smooth part of $\hat{A}$ and $\hat{\alpha}=f_{*} \alpha$ there. Due to the relation (R2), such a pair $(\hat{A}, \hat{\alpha})$ carries precisely the same information as $(A, f, \alpha) \cdot{ }^{1}$ (The only point to worry about is

\footnotetext{
${ }^{1}$ Note, that the consideration of triples $(A, f, \alpha)$ instead of pairs $(\hat{A}, \hat{\alpha})$, which we used in Sect. 1 , is similar to the definition of chains in the singular homology theory: in the latter case, although one considers the mappings of abstract simplices into the manifold, but morally it is only "images of simplices" that matter. Here comes also an important distinction: unlike the topological homology, where in each dimension $k$ one uses all continuous maps of one standard object (the standard $k$-simplex or the standard $k$-cell) to a given topological space, in polar homology we deal with complex analytic maps of a large class of $k$-dimensional varieties to a given one.
} 
that such pairs cannot be arbitrary. In fact, by the Hironaka theorem on resolution of singularities, any subvariety $\hat{A} \subset X$ can be the image of some regular $A$, but the form $\hat{\alpha}$ on the smooth part of $\hat{A}$ cannot be arbitrary.)

Definition 2.6 The boundary operator $\partial: \mathcal{C}_{k}(X) \rightarrow \mathcal{C}_{k-1}(X)$ is defined by

$$
\partial(A, f, \alpha)=2 \pi i \sum_{i}\left(V_{i}, f_{i}, \operatorname{res}_{V_{i}} \alpha\right)
$$

(and by linearity), where $V_{i}$ are the components of the polar divisor of $\alpha$, $\operatorname{div}_{\infty} \alpha=\cup_{i} V_{i}$, and the maps $f_{i}=\left.f\right|_{V_{i}}$ are restrictions of the map $f$ to each component of the divisor.

Theorem 2.7 The boundary operator $\partial$ is well defined, i.e. it is compatible with the relations ( $\mathrm{R} 1),(\mathrm{R} 2),(\mathrm{R} 3)$.

Proof. We have to show that $\partial$ maps equivalent sums of triples to equivalent ones.

It is trivial with (R1). For (R2) this follows from the commutativity of taking residue and push-forward. To prove the compatibility of $\partial$ with (R3) consider first the case of a polar 1-chain, a complex curve with a meromorphic 1-form, which is mapped to a point.

Then the image of the boundary of this 1-chain is zero. Indeed, this image must be the same point, whose coefficient is equal to the sum of all residues of the meromorphic 1-form on the curve, i.e. zero. The general case is similar: the same phenomenon occurs along one of the coordinates.

Theorem 2.8 $\quad \partial^{2}=0$.

Proof. We need to prove this for triples $(A, f, \alpha) \in \mathcal{C}_{k}(X)$, i.e., for forms $\alpha$ with normal crossing divisors of poles. The repeated residue at pairwise intersections differs by a sign according to the order in which the residues are taken, see 2.2. Thus the contributions to the repeated residue from different components cancel out (or, the residue of a residue is zero)..$^{2}$

\section{2c. Polar homology of projective varieties.}

Definition 2.9 For a complex projective variety $X, \operatorname{dim} X=n$, the chain complex

$$
0 \rightarrow \mathcal{C}_{n}(X) \stackrel{\partial}{\longrightarrow} \mathcal{C}_{n-1}(X) \stackrel{\partial}{\longrightarrow} \cdots \stackrel{\partial}{\longrightarrow} \mathcal{C}_{0}(X) \rightarrow 0
$$

is called the polar chain complex of $X$. Its homology groups, $H P_{k}(X), k=0, \ldots, n$, are called the polar homology groups of $X$.

\footnotetext{
${ }^{2}$ One can note that an example of the polar divisor $\{x y=0\}$ for the form $d x \wedge d y / x y$ in $\mathbb{C}^{2}$ should be viewed as a complexification of a polygon vertex in $\mathbb{R}^{2}$. Indeed, the cancellation of the repeated residues on different components of the divisor is mimicking the calculation of the boundary of a boundary of a polygon: every polygon vertex appears twice with different signs as a boundary point of two sides.
} 
Functoriality of polar homology is standard: A regular morphism of projective varieties $h: X \rightarrow Y$ defines a homomorphism $h_{*}: P H_{k}(X) \rightarrow P H_{k}(Y){ }^{3}$

2.10 EXAMPLE. For a projective curve of genus $g$ the polar homology groups are as follows: $H P_{0}=\mathbb{C}, H P_{1}=\mathbb{C}^{g}$, and $H P_{k}=0$ for $k \geq 2$. One can readily see that the approach with triples coincides with the consideration of Introduction.

2.11 Relative polar homology. Let $Z$ be a projective subvariety in a projective $X$. Analogously to the topological relative homology we can define the polar relative homology of the pair $Z \subset X$.

Definition 2.12 The relative polar homology $H P_{k}(X, Z)$ is the homology of the following quotient complex of chains:

$$
\mathcal{C}_{k}(X, Z)=\mathcal{C}_{k}(X) / \mathcal{C}_{k}(Z)
$$

Here we use the natural embedding of the chain groups $\mathcal{C}_{k}(Z) \hookrightarrow \mathcal{C}_{k}(X)$. This leads to the long exact sequence in polar homology:

$$
\ldots \rightarrow H P_{k}(X) \rightarrow H P_{k}(X, Z) \stackrel{\partial}{\longrightarrow} H P_{k-1}(Z) \rightarrow H P_{k-1}(X) \rightarrow \ldots
$$

\section{Dolbeault cohomology as polar de Rham cohomology.}

As we discussed in the Introduction, the Dolbeault complex of $(0, k)$-forms should be related to the polar homology in the same way as the de Rham complex of smooth forms is related to the topological homology (e.g., singular homology). Now, after the definitions of Sect. 2 are given, we are able to make this point more explicit.

3.1 In a smooth projective variety $X$, consider a polar $k$-chain, for instance, a prime one, i.e. (an equivalence class of) a triple $a=(A, f, \alpha)$. Such a triple can be regarded as a linear functional on the space of smooth $(0, k)$-forms on $X$. (In other words, the space of polar chains $\mathcal{C}_{k}(X)$ can be defined as a subspace of currents, functionals on smooth differential forms.) Let $u$ be a smooth $(0, k)$-form on $X$, then the pairing is given by the following integral:

$$
\langle a, u\rangle:=\int_{A} \alpha \wedge f^{*} u
$$

The integral is well defined since $\alpha$ has only first order poles on a normal crossing divisor. It is now straightforward to show that the pairing $\langle$,$\rangle descends to the space of$ equivalence classes of triples $\mathcal{C}_{k}(X)$, i.e., that it is compatible with the relations $(\mathrm{R} 1)$, (R2), (R3) of Definition 2.4. Indeed, (R1) is obvious, compatibility with (R3) follows from noticing that $f^{*} u=0$ if $\operatorname{dim} f(A)<k$, and the compatibility with (R2) follows from the relation $\int_{A} \alpha \wedge f^{*} u=\int_{f(A)} f_{*} \alpha \wedge u$ if $\operatorname{dim} f(A)=k$, where the last integral is taken over the smooth part of $f(A)$.

\footnotetext{
${ }^{3}$ Note that the polar homology is an analogue of the non-torsion part of singular homology, the homology groups with real coefficients.
} 
Proposition 3.2 The pairing (3.1) defines the following homomorphism in (co)homology:

$$
\rho: H P_{k}(X) \rightarrow H_{\bar{\partial}}^{n, n-k}(X)
$$

where $n=\operatorname{dim} X$.

Proof. By the Serre duality, $\rho$ is the map $H P_{k}(X) \rightarrow\left(H_{\bar{\partial}_{\bar{D}}}^{0, k}(X)\right)^{*}$ and it is sufficient to verify that the pairing (3.1) vanishes if $\partial a=0$ and $u=\bar{\partial} v$, or if $\bar{\partial} u=0$ and $a=\partial b$. This follows immediately from the Cauchy-Stokes formula (1.5):

$$
\int_{A} \alpha \wedge f^{*}(\bar{\partial} u)=2 \pi i \int_{\operatorname{div}_{\infty} \alpha}(\operatorname{res} \alpha) \wedge f^{*}(u)
$$

that is $\langle a, \bar{\partial} u\rangle=\langle\partial a, u\rangle$.

It turns out that for smooth projective manifolds the homomorphism (3.2) is in fact an isomorphism.

Theorem 3.3 (Polar de Rham theorem, [KRT])

a) For a smooth projective manifold $X$ the mapping $\rho: H P_{k}(X) \rightarrow H_{\bar{\partial}}^{n, n-k}(X)$ is an isomorphism of the polar homology and Dolbeault cohomology groups. Equivalently, in terms of dual cohomology groups,

$$
H P^{k}(X) \cong H_{\bar{\partial}}^{0, k}(X)
$$

b) Let $V$ be a normal crossing divisor in a smooth projective $X$. Then

$$
H P_{q}(X, V) \simeq H^{n-q}\left(X, K_{X}(V)\right)
$$

3.4 EXAMPLE. If $X$ is a complex curve of genus $g$ one has $H P_{0}(X) \cong \mathbb{C} \cong H_{\bar{\partial}}^{1,1}(X)$ and $H P_{1}(X) \cong \mathbb{C}^{g} \cong H_{\bar{\partial}}^{1,0}(X)$ (see Example 1.4).

Note that by Theorem 3.3, for an ample divisor $V$ represented by a smooth hypersurface in $X, H P_{p}(X, V)=H^{n-p}\left(X, K_{X}(V)\right)=0$. Such a choice of the hypersurface $V$ in the complex manifold $X$ is similar to the choice of an $(n-1)$-skeleton in an $n$-dimensional real manifold: the corresponding relative homology group of the manifold with respect to the skeleton also vanish.

3.5 REMARK. So far we considered polar chains with top degree forms. More generally, one could consider polar $(k, p)$-chains $(A, f, \alpha)$, where $\alpha$ is a meromorphic $p$-form of not necessarily maximal degree, $p \leqslant k$, on $A$ that can have only logarithmic singularities on a normal crossing divisor. ${ }^{4}$ The requirement of log-singularities is needed to have a convenient definition of the residue and, hence, the boundary operator $\partial$.

The property $\partial^{2}=0$ and the definition of the polar homology groups can be carried over to this, more general, situation. The polar homology groups are then enumerated by two indices: $H P_{k, p}(M)$.

\footnotetext{
${ }^{4} \mathrm{An}$ important property of such forms on projective varieties is that they are closed, see [De].
} 
The Cauchy-Stokes formula (1.5) extends to this case as well. As a consequence, the natural pairing between polar $(k, p)$-chains and smooth $(k-p, k)$-forms on $X$ gives us as before the homomorphism (cf. (3.2))

$$
\rho: H P_{k, p}(X) \rightarrow H_{\bar{\partial}}^{n-k+p, n-k}(X) .
$$

However, unlike the case $p=k$, the map $\rho$ is not, in general, an isomorphism for other values of $p, 0 \leqslant p<k$. For instance, in the case of $p=0$, the image consists of algebraic

$r$-cycles (tensored with $\mathbb{C}$ ), while the full $H_{\bar{\partial}}^{r, r}(X)$ can be much larger (already for a generic K3 surface).

One can see that for $p=0$ the polar homology groups $H P_{k, 0}(X)$ coincide with the groups of $k$-cycles in $X$ modulo algebraic equivalence, according to the Severi theorem, [GH]. Thus the groups $H P_{k, p}(X)$ interpolate between the groups of cycles modulo algebraic equivalence and the Dolbeault groups as $p$ changes from 0 to $k$.

\section{Intersection in polar homology.}

We define here a polar analogue of the topological intersection product. In particular, for polar cycles of complimentary dimensions one obtains a complex number, called the polar intersection number.

Recall that in topology, one considers a smooth oriented closed manifold $M$ and two oriented closed submanifolds $A, B \subset M$ of complementary dimensions, i.e., $\operatorname{dim} A+$ $\operatorname{dim} B=\operatorname{dim} M$. Suppose, $A$ and $B$ intersect transversely at a finite set of points. Then to each intersection point $P$ one assigns \pm 1 (local intersection index) by comparing the mutual orientations of the tangent vector spaces $T_{P} A, T_{P} B$, and $T_{P} M$.

\section{4a. Polar oriented manifolds.}

Let now $M$ be a compact complex manifold of dimension $n$, on which we would like to define a polar intersection theory. It has to be polar oriented, i.e., equipped with a complex volume form. As the discussion below shows, the $n$-form $\mu$ defining its polar orientation has to have no zeros on $M$, since we are going to consider expressions in which $\mu$, the orientation of the ambient manifold, enters a denominator. Therefore we adopt the following terminology.

Definition 4.1 i) A compact complex manifold $M$, endowed with a nowhere vanishing holomorphic volume form $\mu$, is said to be a polar oriented closed manifold.

ii) If the volume form $\mu$ on a compact complex manifold $M$ is nonvanishing and meromorphic with only first order poles on a normal crossing divisor $N \subset M$, then $M$ is called a polar oriented manifold with boundary. The hypersurface $N$ is then endowed with a polar orientation $\nu:=2 \pi i$ res $\mu \neq 0$ and $(N, \nu)$ is called the polar boundary of $(M, \mu)$. 
4.2 Remark. By definition, polar orientable closed manifolds are complex manifolds whose canonical bundle is trivial (Calabi-Yau, Abelian manifolds or, for example, any complex tori, if we do not restrict ourselves to algebraic manifolds). We have just defined the notion of the polar orientation in a more restrictive sense than before, when we considered the definition of chains. In fact, polar chains with their orientations are to be compared to oriented piece-wise smooth submanifolds in differential topology, while the ambient space on which we want to have Poincaré duality has to be everywhere smooth and oriented. Zeros of a volume form could be regarded as a complex analogue of singularities of a real manifold. ${ }^{5}$

\section{4b. Polar intersection number.}

4.3 Let $(M, \mu)$ be a polar oriented closed manifold of dimension $n$. In such a case we define the following natural pairing between its polar homology groups $H P_{p}(M)$ and $H P_{q}(M)$ of complimentary dimension $(p+q=n)$.

First we define the polar intersection number for the case of a smooth and transverse pair of cycles $a$ and $b$. That is $a=(A, \alpha)$ and $b=(B, \beta)$, where $A$ is a smooth $p$ dimensional subvariety and $\alpha$ a holomorphic $p$-form on it (and similarly for $(B, \beta)$ in dimension $q$ ) and it is assumed that $A$ and $B$ intersect transversely. Then, we have the following formula for the polar intersection index.

Definition 4.4 The polar intersection index of two smooth transverse cycles $(A, \alpha)$ and $(B, \beta)$ is given by the following sum over the set of points in $A \cap B$ :

$$
(A, \alpha) \cdot{ }_{\mu}(B, \beta)=\sum_{P \in A \cap B} \frac{\alpha(P) \wedge \beta(P)}{\mu(P)}
$$

Here $\alpha(P)$ and $\beta(P)$ are understood as exterior forms on $T_{P} M=T_{P} A \times T_{P} B$ obtained by the pull-back from the corresponding factors.

The ratio in the right-hand-side can be understood as the comparison of the polar orientations brought to the intersection point $P$ by the two cycles with the polar orientation $\mu(P)$ of the ambient manifold at that point. Recall, that on a polar oriented closed manifold the holomorphic $n$-form $\mu$ vanishes nowhere.

To extend this definition to arbitrary and not necessarily transverse polar cycles we apply the following

Proposition 4.5 For any two polar cycles $a$ and $b$ there exist polar homologous cycles $a^{\prime}$ and $b^{\prime}$ which are represented by smooth transversely intersecting submanifolds.

\footnotetext{
${ }^{5}$ For instance, on a complex curve $X$ of genus $g$ one has $H P_{1}(X)=\mathbb{C}^{g}$, and a holomorphic 1differential representing a generic element in $H P_{1}(X)$ has $2 g-2$ zeros. From this point of view the complex genus $g$ curve is like a graph which has $g$ loops joined by $g-1$ edges and having $2 g-2$ trivalent (i.e., "non-smooth") points. The "smooth orientable cases" are $\mathbb{C P}^{1}$, which corresponds to a real segment, and an elliptic curve, which is a complex counterpart of the circle in this precise sense.
} 
Proof. By Theorem 3.3, if $V$ is a normal crossing divisor in $M$, then $\operatorname{HP}_{p}(M, V)=$ $H^{n-p}\left(M, K_{M}(V)\right)$. Choose $V$ to be an ample divisor represented by a smooth hypersurface. Then the right hand side is 0 , and so is $H P_{p}(M, V)=0$. This shows that any polar cycle is homologous to a cycle lying in an ample smooth hypersurface $V$. Iterating this consideration inside $V$ we arrive at the fact that any polar cycle is homologous to a smooth one. An analogous reasoning shows that one can achieve a transversal intersection for two such cycles.

It turns out that the definition of polar intersection extends to the classes of polar homologous cycles.

Theorem 4.6 The polar intersection is a well-defined pairing in polar homology.

To define the polar intersection pairing one can compose the homomorphism $\rho$ to and the product in the Dolbeault cohomology. Namely, according to Proposition 3.2, the groups $H P_{q}(M)$ and $H P_{p}(M)$ can be mapped isomorphically to the Dolbeault cohomology groups $H_{\bar{\partial}}^{n, n-q}(M)$ and $H_{\bar{\partial}}^{n, n-p}(M)$, respectively. On a polar oriented closed manifold we are given a nowhere vanishing section $\mu$ of the line bundle $K_{M}$. Hence, we have the isomorphism $H_{\bar{\partial}}^{n, n-q}(M) \stackrel{\mu^{-1}}{\longrightarrow} H_{\bar{\partial}}^{0, n-q}(M)$. Using this and the product in Dolbeault cohomology we obtain the following pairing for $p+q=n$ :

$$
H_{\bar{\partial}}^{n, n-p}(M) \otimes H_{\bar{\partial}}^{n, n-q}(M) \stackrel{\operatorname{id} \otimes \mu^{-1}}{\longrightarrow} H_{\bar{\partial}}^{n, n-p}(M) \otimes H_{\bar{\partial}}^{0, n-q}(M) \rightarrow H_{\bar{\partial}}^{n, n}(M) \stackrel{\sim}{\rightarrow} \mathbb{C} .
$$

One can see that this definition of pairing coincides with the one given in Definition 4.4 for transverse representatives. As a byproduct, one has the following non-degeneracy theorem.

Corollary 4.7 The polar intersection pairing is non-degenerate, as a bilinear form on the polar homology groups

$$
H P_{p}(M) \otimes H P_{q}(M) \rightarrow \mathbb{C}
$$

where $p+q=n$.

\section{4c. Polar intersection product.}

Now consider the case when on a polar oriented closed manifold $(M, \mu)$ we have two polar cycles of arbitrary dimensions $p$ and $q$ (not necessarily complimentary ones). For any $p$ and $q$ one can define an intersection product in polar homology.

4.8 To describe this intersection product on two smooth transverse cycles we need the following construction from the linear algebra for $\mathbb{C}$-oriented vector spaces. Let $V_{A}, V_{B} \subset$ $W$ be two transverse vector subspaces of dimensions $p$ and $q$, respectively, in a complex $n$-dimensional vector space $W(p+q \geqslant n)$. Suppose we are given "CC-orientations" of all these spaces, i.e., the (non-zero) exterior forms $\alpha_{0} \in \bigwedge^{p} V_{A}^{*}, \beta_{0} \in \bigwedge^{q} V_{B}^{*}$, and $\mu_{0} \in \bigwedge^{n} W^{*}$. 
Then the intersection $V_{A} \cap V_{B}$ can be naturally endowed with a $\mathbb{C}$-orientation, i.e., the exterior form

$$
\gamma_{0}=\frac{\alpha_{0} \wedge \beta_{0}}{\mu_{0}}
$$

This notation stands for the following. In coordinate form, we can always choose the coordinates $z_{1}, \ldots, z_{n}$ in $W$ such that the space $V_{A}$ is spanned by $z_{1}, \ldots, z_{p}$ and $V_{B}$ is spanned by $z_{n-q+1}, \ldots, z_{n}$, while $\alpha=d z_{1} \wedge \cdots \wedge d z_{p}$ and $\beta=d z_{n-q+1} \wedge \cdots \wedge d z_{n}$. Then for $\mu_{0}=c_{0} \cdot d z_{1} \wedge \cdots \wedge d z_{n}$ one has

$$
\gamma_{0}:=\frac{d z_{n-q+1} \wedge \cdots \wedge d z_{p}}{c_{0}}
$$

One readily verifies that $\gamma_{0}$ does not depend on a coordinate choice (see $[K R]$ ).

Now one can define the polar orientation for intersection of polar cycles.

Definition 4.9 For a pair of two smooth transverse polar cycles $a=(A, \alpha)$ and $b=$ $(B, \beta)$ of dimensions $p$ and $q$, respectively, in a polar oriented closed manifold $(M, \mu)$, their intersection is a polar $(p+q-n)$-cycle $c=(C, \gamma)$, where $C=A \cap B$ and

$$
\gamma=\frac{\alpha \wedge \beta}{\mu} \text {. }
$$

[Here the definition of the holomorphic form $\gamma$ on $C$ is given by the linear algebra from Section 4.8 above at every point of $C$.]

Similarly to the $p+q=n$ case (Definition 4.4 ), the intersection of smooth transverse cycles

$$
(A, \alpha) \cdot{ }_{\mu}(B, \beta)=(C, \gamma)
$$

for $p+q>n$ defines the intersection in polar homology

$$
H P_{p}(M) \otimes H P_{q}(M) \rightarrow H P_{p+q-n}(M),
$$

upon finding smooth transverse representatives for every pair of homology classes. One can see that the intersection is well-defined in the following formulation for $0 \leqslant p+q \leqslant n$ :

$$
\begin{gathered}
H_{\bar{\partial}}^{n, n-p}(M) \otimes H_{\bar{\partial}}^{n, n-q}(M) \stackrel{{ }^{\mathrm{id} \otimes \mu^{-1}}(M) \otimes H P_{q}(M) \stackrel{\sim}{\rightarrow}}{\longrightarrow} H_{\bar{\partial}}^{n, n-p}(M) \otimes H_{\bar{\partial}}^{0, n-q}(M) \rightarrow H_{\bar{\partial}}^{n, 2 n-p-q}(M) \\
\stackrel{\sim}{\rightarrow} H P_{p+q-n}(M) .
\end{gathered}
$$

4.10 Remark. We have defined the polar intersection on any complex manifold $M$ that can be equipped with a holomorphic non-vanishing volume form $\mu$. This is analogous to the topological intersection theory on a compact smooth oriented manifold without boundary. (Note that the Poincaré duality in this context should correspond to the Serre duality, cf. [DT, Th].) Furthermore, the consideration above easily extends to the case of a complex manifold possessing a meromorphic non-vanishing form $\mu$ (in particular, to a complex projective space), i.e., to the case of a polar oriented manifold $(M, \mu)$ with boundary $(N, 2 \pi i$ res $\mu$ ) (cf. 4.1$)$. The latter setting is similar to the topological intersection theory on manifolds with boundary. In this case the above formulas can be used to define the pairing between polar homology $H P_{k}(M)$ and polar homology relative to the boundary $H P_{n-k}(M, N)$. 


\section{$5 \quad$ Polar linking number and Weil pairing.}

Recall that the Gauss linking number of two oriented closed curves in $\mathbb{R}^{3}$ is an integer topological invariant equal to the algebraic number of crossings of one curve with a two-dimensional oriented surface bounded by the other curve. The independence of the linking number on the choice of the surface follows from the fact that the algebraic number of intersections of a closed curve and a closed surface in a simply connected three-dimensional manifold is equal to zero. Note that the Gauss linking number is a homology invariant in the following sense: it does not change if one of the curves is replaced by a homologically equivalent cycle in the compliment to the other curve. More generally, the linking number can be defined for two oriented closed submanifolds of linking dimensions in any oriented (but not necessarily simply connected) manifold, provided that both submanifolds are homologous to zero.

\section{5a. Definition of polar linking number.}

Polar linkings mimic the classical definition of linking in the polar language. Let $a=(A, \alpha)$ and $b=(B, \beta)$ be two polar smooth non-intersecting cycles of dimensions $p$ and $q$ in a polar oriented closed $n$-manifold $(M, \mu)$. Suppose that these cycles are polar boundaries (i.e. they are polar homologous to 0 ) and are of linking dimensions: $p+q=n-1$. Then one can associate to them the following polar linking number.

Definition 5.1 The polar linking number of cycles $a$ and $b$ in $(M, \mu)$ is

$$
\ell k_{\mu}(a, b):=\sum_{P \in A \cap S} \frac{\alpha(P) \wedge \sigma(P)}{\mu(P)}
$$

where a chain $(S, \sigma)$ has the polar boundary $(B, \beta), \partial(S, \sigma)=(B, \beta)$.

In other words, $\ell k_{\mu}(a, b)$ is the intersection of the polar cycle $a$ and the polar chain $s=(S, \sigma)$, provided they intersect transversely.

\section{5b. Properties of polar linking number.}

The definition of polar linking is analogous to the topological one. It also mimics the properties of the latter:

i) The polar linking is (anti-)symmetric:

$$
\ell k_{\mu}(a, b)=(-1)^{(n-p)(n-q)} \ell k_{\mu}(b, a) .
$$

ii) It is well-defined, i.e., $\ell k_{\mu}$ does not depend on the choice of the polar chain $s=(S, \sigma)$, provided that $\partial(S, \sigma)=(B, \beta)$.

iii) If $M$ is a polar manifold with (polar) boundary, there exists a relative version of $\ell k_{\mu}$, as in topology. 
The simplest curves that can have non-trivial linking are elliptic curves. The linking number of a rational curve with any other curve is zero since any holomorphic differential on a rational curve must vanish. (As we discussed before, a rational curve $\mathbb{C} P^{1}$ equipped with a meromorphic 1-form with two simple poles is a complexification of a segment, while an elliptic curve with a holomorphic 1-form on it is an analogue of a circle.)

Similarly to the Gauss formula for the topological linking number, the polar linking can be defined via integrals involving the Green function for $\bar{\partial}$, cf. [FT, Th]. Note that such a Green function is a propagator in the corresponding quantum field theory, and its appearence in the polar linking is natural within the QFT framework.

Note also that the polar linking is an invariant of the corresponding polar homology class $[a] \in H P^{p}(M \backslash B)$. To formulate this property precisely, one needs a definition of the polar homology of complex quasi-projective manifolds and we are going to describe it elsewhere.

\section{5c. Intersections with various coefficients.}

Now we would like to extend the notion of polar chains and cycles, to include the chains $(A, \alpha)$, where $\alpha$ can be not only a logarithmic differential form on a subvariety $A \subset M$, but also, e.g., a meromorphic section of the normal bundle to $A$.

With this more general understanding of polar cycles, one does not need to fix the non-vanishing form $\mu$ in the ambient manifold $M$ (and hence to confine oneself to the class of polar oriented closed manifolds) to define the polar intersection theory.

One can define the polar intersection index of two smooth transverse cycles $(A, \alpha)$ and $(B, \beta)$ in $M$ by

$$
(A, \alpha) \cdot(B, \beta)=\sum_{P \in A \cap B}\langle\alpha(P), \beta(P)\rangle
$$

if the "framings" $\alpha$ and $\beta$ at points $P$ assume values in dual spaces. In other words, we consider the polar chains with appropriate coefficients, and $\langle$,$\rangle is the corresponding$ pairing. Recall that the polar intersection

$$
(A, \tilde{\alpha}) \cdot{ }_{\mu}(B, \tilde{\beta})=\sum_{P \in A \cap B} \frac{\tilde{\alpha}(P) \wedge \tilde{\beta}(P)}{\mu(P)}
$$

of polar $p$ - and $q$-cycles $(A, \tilde{\alpha})$ and $(B, \tilde{\beta})$ in $(M, \mu)$ (where $p+q=n$ ) discussed in the preceding section corresponds to the one just described as follows: $\alpha=\tilde{\alpha}$ is a $p$-form on $A$, while $\beta=\tilde{\beta} / \mu$ is a ratio of an $(n-p)$-form on $B$ and an $n$-form on $M$, i.e. a meromorphic section of $\Lambda^{p} N B$, the $p$-th power of the normal bundle to $B$, or, roughly speaking, a $p$-vector field normal to $B$ in $M$. The latter can be contracted with the $p$-form on $A$ at the intersection points $A \cap B$.

\section{5d. Linkings with other coefficients.}

Similarly, one can define the polar linking of more general polar boundaries "with coefficients": after passing to a polar chain bounded by one of the cycles, this chain and 
the other cycle have to be equipped with appropriate sections assuming values in dual spaces.

5.2 ExAMPLE. Let $a$ and $b^{\prime}$ be 0 -boundaries with certain coefficients on a complex curve $X$. Namely, $a=\sum\left(P_{i}, r_{i}\right)$ is a 0 -cycle, where $P_{i} \in X$ are points with complex coefficients $r_{i} \in \mathbb{C}$, satisfying the exactness condition: $\sum r_{i}=0$, ensuring that $a$ is the boundary of a 1-chain. The cycle $b^{\prime}=\sum\left(Q_{j}, v_{j}\right)$ is a set of points $Q_{j} \in X$ with vectors $v_{j} \in T_{Q_{j}} X$ assigned to them. (We discuss below the restriction on $b^{\prime}$ imposed by the condition of 0-boundary.)

In this setting the following polar linking number of $a$ and $b^{\prime}$ is defined. Let $a=\partial c$, where a polar 1-chain $c=(X, \alpha)$ is such that the 1 -form $\alpha$ has poles of the first order at $P_{i}$ and satisfies $2 \pi i \operatorname{res}_{P_{i}} \alpha=r_{i}$. Then

$$
\ell k_{\text {polar }}\left(b^{\prime}, a\right):=\sum_{j} \iota_{v_{j}} \alpha\left(Q_{j}\right) .
$$

It turns out that this linking number does not depend (up to a sign) on for which of the two 0-boundaries, $a$ or $b^{\prime}$, we use a spanning polar 1-chain. The fact that the 0 -cycle $b^{\prime}$ is a polar boundary means that there exists a function $\phi$ with poles of the first order at $Q_{j}$, such that $2 \pi i \operatorname{res}_{Q_{j}} \phi=v_{j}$. (Note that the Poincaré residue of a function is a vector attached at its pole.) Then $b^{\prime}=\partial(X, \phi)$.

One can see that the linking number $\ell k_{\text {polar }}\left(b^{\prime}, a\right)$ evaluated with the help of the chain $(X, \phi)$ bounding $b^{\prime}$ (rather than $a$ ) leads to the same result. Indeed, consider the 1-form $\phi \alpha$ on $X$. It has poles where so does either the function $\phi$, or the form $\alpha$. Denote by $\Gamma$ the contour encompassing only the poles of $\alpha$. Then

$$
\sum_{i} \phi\left(P_{i}\right) \cdot r_{i}=2 \pi i \sum_{i} \operatorname{res}_{P_{i}}(\phi \cdot \alpha)=\oint_{\Gamma} \phi \cdot \alpha=-2 \pi i \sum_{j} \operatorname{res}_{Q_{j}}(\phi \cdot \alpha)=-\sum_{j} \iota_{v_{j}} \alpha\left(Q_{j}\right) .
$$

Note also that the vector field $v_{j}$ attached to $Q_{j}$ can be regarded as an infinitesimal deformation of the divisor $Q_{j}$. We are going to exploit this point of view below.

\section{5e. The Weil pairing and reciprocity law.}

Let $f$ and $g$ be two meromorphic functions on $X$ with disjoint divisors: $\operatorname{div} f=$ $\sum_{i} r_{i} P_{i}$ and $\operatorname{div} g=\sum_{j} q_{j} S_{j}, \quad$ where $P_{i}, S_{j} \in X$ while $r_{i}=\operatorname{deg}_{P_{i}} f$ and $q_{j}:=\operatorname{deg}_{S_{j}} g$ are integers.

Definition 5.3 The Weil pairing of functions $f$ and $g$ is $\{f, g\}=\prod_{j} f\left(S_{j}\right)^{\operatorname{deg}_{S_{j}} g}$.

The Weil reciprocity law is the symmetry of this bracket:

$$
\{f, g\}=\{g, f\} .
$$

It follows from the identity

$$
\oint_{\Gamma} \log f \cdot d \log g=-\oint_{\Gamma} \log g \cdot d \log f
$$


applied to a contour $\Gamma$ embracing $\operatorname{div} g$ and leaving $\operatorname{div} f$ outside, and using the identity $\operatorname{deg}_{P} g=\operatorname{res}_{P}(d \log g)$, for all points $P \in X$.

\section{5f. Relation of the Weil pairing and polar linking.}

Let $f_{t}$ and $g_{t}$ be one-parameter families of meromorphic functions on $X$ with disjoint divisors $a_{t}:=2 \pi i \operatorname{div} f_{t}$ and $b_{t}:=2 \pi i \operatorname{div} g_{t}$ for all $t$. Note that the divisors $a_{t}$ and $b_{t}$ define polar 0-cycles and these are, in fact, polar 0-boundaries: $a_{t}=\partial(X, \alpha)$, where $\alpha=d f_{t} / f_{t}$, and similarly for $b_{t}$.

Consider the infinitesimal deformation $a_{t}^{\prime}$ (respectively, $b_{t}^{\prime}$ ), i.e., the derivatives in $t$ of $a_{t}$ (respectively, $\left.b_{t}\right)$, which can be defined as follows: $a_{t}^{\prime}=\partial\left(X, \phi_{t}\right)$, where $\phi_{t}:=\frac{d}{d t} \log f_{t}$ is a meromorphic function on $X$. We suppose that $\phi_{t}$ has only simple poles, so that $\left(X, \phi_{t}\right)$ is a polar chain. Note that $a_{t}^{\prime}$ and $b_{t}^{\prime}$ are polar 0-boundaries "with coefficients": a set of points with certain vectors attached.

Proposition 5.4 The following relation between the Weil pairing and the polar linking holds:

$$
2 \pi i \frac{d}{d t} \log \left\{f_{t}, g_{t}\right\}=\ell k_{\text {polar }}\left(a_{t}^{\prime}, b_{t}\right)+\ell k_{\text {polar }}\left(b_{t}^{\prime}, a_{t}\right) \text {. }
$$

Proof. Indeed,

$2 \pi i \frac{d}{d t} \log \left\{f_{t}, g_{t}\right\}=\oint_{\Gamma} \frac{d}{d t}\left(\log f_{t}\right) \cdot \frac{d g_{t}}{g_{t}}-\oint_{\Gamma} \frac{d}{d t}\left(\log g_{t}\right) \cdot \frac{d f_{t}}{f_{t}}=\ell k_{\text {polar }}\left(a_{t}^{\prime}, b_{t}\right)+\ell k_{\text {polar }}\left(b_{t}^{\prime}, a_{t}\right)$.

Here we used that

$$
\oint_{\Gamma} \frac{d}{d t}\left(\log f_{t}\right) \cdot \frac{d g_{t}}{g_{t}}=\oint_{\Gamma} \phi_{t} \cdot \frac{d g_{t}}{g_{t}}=\ell k_{\text {polar }}\left(a_{t}^{\prime}, b_{t}\right)
$$

and similarly for $\ell k_{\text {polar }}\left(b_{t}^{\prime}, a_{t}\right)$.

\section{5g. Higher-dimensional generalization and Parshin symbols.}

On an $(n-1)$-dimensional complex manifold $X$ consider $n$ functions $f_{1}, \ldots, f_{n}$ whose poles and zeros are of the first order. Assume also that their poles and zeros divisors are normal crossing and in general position. Define the bracket $\left\{f_{1}, \ldots, f_{n}\right\}$, having symmetry properties similar to the Weil pairing.

Definition 5.5 For meromorphic functions $f_{1}, \ldots, f_{n}$ on $X$ define the following bracket:

$$
\left\{f_{1}, \ldots, f_{n}\right\}:=\prod_{P} f_{1}(P)^{d_{P}\left(f_{2}, \ldots, f_{n}\right)}
$$

where the product is taken over all points $P$ belonging to the intersection of the divisors $\left|\operatorname{div} f_{2}\right| \cap \cdots \cap\left|\operatorname{div} f_{n}\right|$. If $P \in D_{2} \cap \cdots \cap D_{n}$, where $D_{i}$ is a component of the divisor $\operatorname{div} f_{i}$ entering with multiplicity $d_{i}$ (which is \pm 1 by the assumption), then $d_{P}\left(f_{2}, \ldots, f_{n}\right):=$ $d_{2} \cdot \ldots \cdot d_{n}$. 
In fact, here we iterate the following procedure: take $\operatorname{div} f_{n}$ and restrict to it the remaining functions $f_{1}, \ldots f_{n-1}$. (For $\operatorname{dim} X=1$ above, we, in fact, considered the restriction of $f=f_{1}$ to the divisor of $f_{2}=g$.) Repeat the procedure until we come to the points and can consider the corresponding Weil pairing.

5.6 REMARK. Note that the brackets $\left\{f_{1}, \ldots, f_{n}\right\}$ can be thought of as the Parshin symbols defined, in general, for meromorphic functions in the presence of a flag of subvarieties, see $[\mathrm{Par}, \mathrm{BM}]$. The symmetry properties of the above brackets in $f_{1}, \ldots, f_{n}$ are a particular case of Parshin's reciprocity laws. For instance, the symmetry in $f_{1}$ and $f_{2}$ is evident as it follows from the Weil reciprocity law in dimension 1 after the restriction to a curve.

\section{5h. Higher-dimensional brackets and higher polar linking.}

To discuss the relation of the bracket (5.5) above and the polar linking of cycles we confine the discussion to the case of $\operatorname{dim} X=3$, though all of what follows can be carried over to $n$ dimensions.

Let $f_{1, t}, \ldots, f_{4, t}$ be meromorphic functions on $X$ as above, depending on a parameter $t$. Define

$$
a_{i, t}:=\partial\left(X, d \log f_{i, t}\right),
$$

an exact 2-cycle on 3-dimensional $X$ with values in integers (0-forms). Its derivative

$$
a_{i, t}^{\prime}:=\partial\left(X, \frac{d}{d t} \log f_{i, t}\right)
$$

is an exact 2-cycle with values in normal vector fields. (Here $\phi_{i, t}=\frac{d}{d t} \log f_{i, t}$ is a meromorphic function, assumed to have simple poles. Its residue is a normal vector field on the corresponding divisor of poles.)

Then the following relation holds:

$$
(2 \pi i)^{3} \frac{d}{d t} \log \left\{f_{1, t}, \ldots, f_{4, t}\right\}=\sum \ell k_{\text {polar }}\left(a_{1}^{\prime}, a_{2} \cdot a_{3} \cdot a_{4}\right),
$$

where the sum is taken over all cyclic permutations. Here $a_{2} \cdot a_{3} \cdot a_{4}$ is the exact polar 0 cycle, obtained by intersecting the corresponding divisors $a_{i}$ and multiplying the numbers on them, which can be linked with the 2-cycle $a_{1}^{\prime}$ in the 3-dimensional $X$.

By definition of $\ell k_{\text {polar }}$ the right-hand-side can also be rewritten as the multiple polar intersection:

$$
\ell k_{\text {polar }}\left(a_{1}^{\prime}, a_{2} \cdot a_{3} \cdot a_{4}\right)=A_{1} \cdot a_{2} \cdot a_{3} \cdot a_{4},
$$

where $\partial A_{1}=a_{1}^{\prime}$. This form can be rewritten, e.g., via pairwise intersections as $\left(A_{1} \cdot a_{2}\right)$. $\left(a_{3} \cdot a_{4}\right)$. The latter product establishes the relation of the bracket (5.5) with the polar linking of 1-cycles:

$$
(2 \pi i)^{3} \frac{d}{d t} \log \left\{f_{1, t}, \ldots, f_{4, t}\right\}=\ell k_{\text {polar }}\left(c_{12}^{\prime}, c_{34}\right)+\ell k_{\text {polar }}\left(c_{34}^{\prime}, c_{12}\right),
$$

where the curves $c_{12}=a_{1} \cdot a_{2}$ and $c_{34}=a_{3} \cdot a_{4}$ are pairwise intersections of the divisors, and, e.g., $c_{12}^{\prime}:=a_{1}^{\prime} \cdot a_{2}+a_{2}^{\prime} \cdot a_{1}$ can be regarded as the $t$-derivative of $c_{12}$. 
5.7 REMARK. The higher polar linking appear as the answers for the correlators of the abelian holomorphic Chern-Simons functional on higher-dimensional complex manifolds and is similar to the topological theory. The most intriguing case is that of a non-abelian holomorphic Chern-Simons theory on a three-dimensional Calabi-Yau manifold, which is expected to produce holomorphic analogs of Vassiliev (finite-order) link invariants.

Acknowledgments. B.K. and A.R. are grateful for hospitality to the RiP program at the Oberwolfach Mathematical Institute, as well as to the Max-Planck-Institut für Mathematik in Bonn and the IHES in Bures-sur-Yvette, where this work was conceived and later completed. We are indebted to S. Donaldson and R. Thomas for discussions on various parts of this paper.

The work of B.K. was partially supported by an NSERC research grant. The work of A.R. was supported in part by RFBR-01-01-00539, INTAS-99-590, FPRMIST-40-052-1-1-1112, and the Grant 00-15-96557 for the support of scientific schools.

\section{References}

[BM] J.-L. Brylinski and D.A. McLaughlin, The geometry of two-dimensional symbols, K-Theory 10:3 (1996), 215-237.

[De] P. Deligne, Théorie de Hodge. II, Inst. Hautes Études Sci. Publ. Math. 40 (1971), $5-57$.

[DT] S.K. Donaldson and R.P. Thomas, Gauge theory in higher dimensions. In "The geometric universe" (Oxford, 1996), Oxford Univ. Press, Oxford (1998) 31-47.

[FK] I.B. Frenkel and B.A. Khesin, Four dimensional realization of two-dimensional current groups, Comm. Math. Phys. 178 (1996), 541-561

[FT] I.B. Frenkel and A.N. Todorov, Complex Counterpart of Chern-Simons-Witten theory and holomorphic linking, preprint (2002)

[GH] P.A. Griffiths and J. Harris, Principles of Algebraic Geometry, Wiley, NY (1978)

[KR] B. Khesin and A. Rosly, Polar homology and holomorphic bundles, Philos. Trans. Roy. Soc. London Ser. A 359 (2001), 1413-1427; Polar homology, (math.AG/0009015)

[KRT] B. Khesin, A. Rosly, and R. Thomas, Polar de Rham theorem, (math.AG/0305081), submitted to Topology.

[Par] A.N. Parshin Residues and duality on algebraic surfaces, Uspehi Mat. Nauk 32:2 (1977), 225-226

[Pol] A.M. Polyakov, Fermi-Bose transmutations induced by gauge fields, Modern Phys. Lett. A 3:3 (1988), 325-328

[Sch] A.S. Schwarz, The partition function of degenerate quadratic functional and RaySinger invariants, Lett. Math. Phys. 2:3 (1977/78), 247-252 
[Th] R.P. Thomas, Gauge theory on Calabi-Yau manifolds, Ph.D. thesis, Oxford (1997), $1-104$

[Wit1] E. Witten, Quantum field theory and the Jones polynomial, Comm. Math. Phys. 121:3 (1989), 351-399

[Wit2] E. Witten, Chern-Simons gauge theory as a string theory, The Floer memorial volume, Progr. Math., 133, Birkhäuser, Basel (1995) 637-678 (hep-th/9207094) 